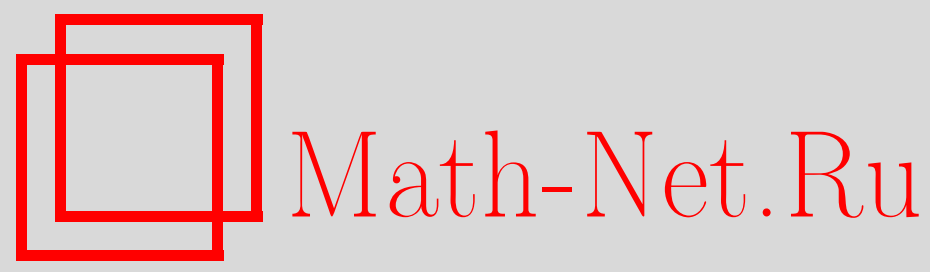

А. Б. Шаповал, Об интегральном многообразии неавтономного эволюционного дифференциального уравнения, $\mathrm{Ma-}$ тем. заметки, 1997, том 61, выпуск 2, 317-320

DOI: https://doi.org/10.4213/mzm1505

Использование Общероссийского математического портала Math-Net.Ru подразумевает, что вы прочитали и согласны с пользовательским соглашением http://www . mathnet.ru/rus/agreement

Параметры загрузки:

IP: 54.237 .206 .68

26 апреля 2023 г., 04:38:01

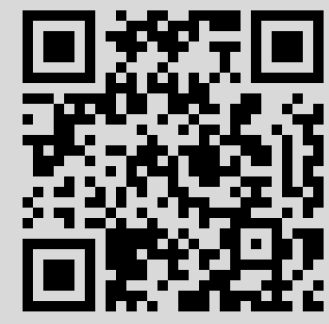




\section{ОБ ИНТЕГРАЛЬНОМ МНОГООБРАЗИИ НЕАВТОНОМНОГО ЭВОЛЮЦИОННОГО ДИФФЕРЕНЦИАЛЬНОГО УРАВНЕНИЯ}

\section{А. Б. Шаповал}

В статье исследуется неавтономное нелинейное уравнение

$$
d_{t} u+A u+f(u, t)=0
$$

в абстрактном гильбертовом пространстве $H$. Предполагается, что $A$ - положительный самосопряженный оператор, обладающий компактным обратным оператором $A^{-1}$. Оператор $A$ удовлетворяет следующему спектральному условию:

$$
\frac{\lambda_{N+1}-\lambda_{N}}{\lambda_{N}^{\beta}+\lambda_{N+1}^{\beta}}>C(f),
$$

где $0<\lambda_{1} \leqslant \lambda_{2} \leqslant \ldots \leqslant \lambda_{N} \leqslant \cdots$ - собственные значения оператора $A ; \beta, 0 \leqslant \beta<1,-$ некоторое фиксированное число, а $C(f)$ - константа, зависящая от $f$. Нелинейньй оператор $f(u, t)$ непрерьвно отображает $D(A) \subset H$ в $D\left(A^{1-\beta}\right)$ и удовлетворяет дополнительным условиям, которые сформулированы в п. 1 .

В работе установлено существование $(N+1)$-мерного интегрального многообразия уравнения (0.1). Число $N$ определяется из условия (0.2). Аналогичные исследования в автономном случае проведены в [1]-[3]. Общая теория изложена в [4].

1. Основные результаты. Предположим, что собственные векторы $\left\{w_{j}\right\}_{j=1}^{\infty}$ оператора $A$ образуют базис в пространстве $H$. Обозначим через $V_{2 \alpha}=D\left(A^{\alpha}\right), \alpha>0$, области определения операторов $A^{\alpha}, V_{2}=V$. Скалярное произведение и норма в пространствах $V_{2 \alpha}$ задаются формулами $(u, v)_{2 \alpha}=\left(A^{\alpha} u, A^{\alpha} v\right)$ и $\|u\|_{2 \alpha}=\left\|A^{\alpha} u\right\|$, где через $(\cdot, \cdot)$ и $\|\cdot\|$ обозначаются скалярное произведение и норма в пространстве $H$. Обозначим через $P_{m}$ ортогональный проектор из $V$ в $V_{m}=\operatorname{span}\left\{w_{1}, \ldots, w_{m}\right\}, Q_{m}=I-P_{m}$.

Предположим, что отображение $f(\cdot, t): V \rightarrow V_{2-2 \beta}$ удовлетворяет следующим условиям:

а) существует производная по $\Phi$ реше $f_{u}^{\prime}(u, t)$ отображения $f$, для которой справедливы неравенства

$$
\begin{aligned}
\left\|f_{u}^{\prime}(u, t) v\right\| \leqslant M_{0}\left(\|u\|_{2}\right)\|v\|_{2 \beta} & \forall u, v \in V, \quad t \in \mathbb{R}, \\
\left\|f_{u}^{\prime}(u, t) v\right\|_{2-2 \beta} \leqslant M_{1}\left(\|u\|_{2}\right)\|v\|_{2} & \forall u, v \in V, \quad t \in \mathbb{R},
\end{aligned}
$$

б) вьполнено

$$
\|(f(u, t+s)-f(u, t))\|_{2-2 \beta} \leqslant M_{2}(\|u\|)|s| \quad \forall u \in V, \quad s \in \mathbb{R},
$$

где $M_{i}(\cdot), i=0,1,2,-$ монотонные функции.

Под решением уравнения (0.1) при начальном условии

$$
\left.u\right|_{t=\tau}=u_{\tau}
$$

понимается функция $u(t) \in C^{1}([\tau, \infty), V)$, удовлетворяющая $(0.1)$ как равенству в $H$. Предположим, что задача $(0.1),(1.4)$ имеет единственное решение при любом $u_{\tau} \in V$. 
Через $\{U(t, \tau), t>\tau\}$ обозначим процесс, соответствующий уравнению $(0.1)$, $U(t, \tau) u(\tau)=u(t)$, где $u(t)$ - решение задачи (0.1), (1.4). Тогда $U(t, \tau): V \rightarrow V$. Предполагается, что процесс $\{U(t, \tau), t>\tau\}$ обладает равномерным по $\tau \in \mathbb{R}$ ограниченньм в $V$ поглощающим множеством (см. [5]), лежащим в шаре с центром в нуле и радиуса $\rho_{0}$. Определим срезающую функцию $q \in C^{1}\left(\mathbb{R}_{+},[0,1]\right)$, обладающую следуюшими свойствами: $q(s)=1$ при $0 \leqslant s \leqslant 1, q(s)=0$ при $s \geqslant 2$ и $\left|q^{\prime}(s)\right| \leqslant 2$. Фиксируем $\rho=2 \rho_{0}$ и положим

$$
F(u, t)=q\left(\frac{\|u\|_{2}^{2}}{\rho^{2}}\right) f(u, t) .
$$

Вместе с уравнением (0.1) рассматривается измененное уравнение

$$
d_{t} u+A u+F(u, t)=0 .
$$

ОПРЕДЕЛЕНИЕ 1.1. Конечномерное липшицево многообразие $M$ будем называть интегральным многообразием уравнения (1.6), если вьполнены следующие условия:

i) если $M(s)$ - сечение множества $M$ в момент времени $s$, то $U(t, s) M(s)=M(t)$, $t>s$

ii) многообразие $M$ экспоненциально притягивает все решения уравнения (1.6).

Теорема 1.1. Пусть выполнены указанные выше условия. Тогда

1) уравнение (1.6) обладает $(N+1)$-мерным интегральным многообразием $M$;

2) для любого решения $и(t)$ задачи $(0.1),(1.4)$ существуют $t_{1} \in[\tau, \infty)$ и решение $v(t) \in M$ уравнения (0.1) такие, что

$$
\|u(t)-v(t)\|_{\beta} \leqslant 2\left\|Q_{N}\left(u\left(t_{1}\right)-v\left(t_{1}\right)\right)\right\|_{\beta} e^{-\mu_{N}\left(t-t_{1}\right)},
$$

әде $\mu_{N}-$ некоторая константа.

2. Построение интегрального многообразия. В этом пункте указаны методы доказательства теоремы 1.1. Интегральное многообразие $M \subset V \times \mathbb{R}$ строится методом Перрона, как график липшицевой функции $\varphi: P_{N} V \times \mathbb{R} \rightarrow Q_{N} V$ :

$$
M=\left\{(p, q, s) \in P_{N} V \times Q_{N} V \times \mathbb{R}: q=\varphi(p, s), T \varphi=\varphi\right\} .
$$

Функция $\varphi$ является неподвижной точкой оператора $T$, который будет определен ниже формулой (2.9).

Обозначим через $\Phi=\Phi_{b, \varkappa, \nu}$ множество функций $\varphi(p, s), \varphi: P_{N} V \times \mathbb{R} \rightarrow Q_{N} V$, удовлетворяющих условиям

$$
\begin{gathered}
\operatorname{supp} \varphi \subset\left\{(p, s): p \in P_{N} V,\|p\|_{2} \leqslant 4 \rho, s \in \mathbb{R}\right\} \\
\left\|\varphi\left(p_{1}, s\right)-\varphi\left(p_{2}, s\right)\right\|_{2} \leqslant \varkappa\left\|p_{1}-p_{2}\right\|_{2} \quad \forall p_{1}, p_{2} \in P_{N} V, \quad s \in \mathbb{R}, \\
\left\|\varphi\left(p, s_{1}\right)-\varphi\left(p, s_{2}\right)\right\|_{2} \leqslant \nu\left|s_{1}-s_{2}\right| \quad \forall p \in P_{N} V, \quad s_{1}, s_{2} \in \mathbb{R}, \\
\|\varphi(p, s)\|_{2} \leqslant b \quad \forall p \in P_{N} V, \quad s \in \mathbb{R} .
\end{gathered}
$$

Определим расстояние в $\Phi$ формулой

$$
\left\|\varphi_{1}-\varphi_{2}\right\|_{\Phi}=\sup _{s \in \mathbb{R}} \sup _{p \in P_{N} V}\left\|\varphi_{1}(p, s)-\varphi_{2}(p, s)\right\|_{2}
$$


Легко видеть,что так определенное пространство $\Phi$ является полньм метрическим пространством.

Зафиксируем $b, \varkappa, \nu$ так, чтобы $b=\rho_{0} / 4, \nu=1, \varkappa=1 / 6$. Для любой функции $\varphi \in \Phi$, $p_{0} \in P_{N} V$ и $s \in \mathbb{R}$ обозначим через $p\left(t ; \varphi, p_{0}, s\right)$ решение задачи

$$
\begin{aligned}
& \frac{d p}{d t}+A p+P_{N} F(p+\varphi(p, t), t)=0, \\
& \left.p\right|_{t=s}=p_{0} .
\end{aligned}
$$

Согласно [3], отображение $P_{N} F(p+\varphi(p, t), t)$ липшицево по $p$, поэтому решение системы (2.6), (2.7) определено для любого $t \in \mathbb{R}$.

Определим оператор $T$, действующий на пространстве $\Phi$, формулой

$$
(T \varphi)\left(p_{0}, s\right)=-\int_{-\infty}^{s} e^{(\tau-s) A Q_{N}} Q_{N} F(u(\tau), \tau) d \tau,
$$

где $u(\tau)=p\left(\tau ; \varphi, p_{0}, s\right)+\varphi\left(p\left(\tau ; \varphi, p_{0}, s\right), \tau\right)$.

ТЕОрема 2.1. Предположим, что для некоторого числа $N$ выполнено условие (0.2). Тогда оператор $T$ действует из $\Phi$ в $\Phi$ и является сжиммющ,м.

ДокАЗАТЕЛЬСТво сводится к получению априорных оценок в пространстве $V$ разности двух решений уравнения (2.6). Близкие методы используются в [3].

ДОКАЗАТЕЛЬСТВО ТЕОРЕМЫ 1.1. Из построения множества $M$ следует, что $M$ является $(N+1)$-мерным многообразием, для которого выполнено свойство і). Докажем, что любое решение $u(t)$ задачи $(0.1),(1.4)$ экспоненциально притягивается к некоторому решению $v(t)$ уравнения (0.1), лежащему на интегральном многообразии $M$. Так как уравнение (0.1) имеет ограниченное в $V$ поглощающее множество, то существует $T>0$ такое, что $\|u(t)\|_{2} \leqslant \rho_{0} \forall t \geqslant \tau+T$. Если для некоторого $t^{\prime} \in[\tau+T, \infty)$ $Q_{N} u\left(t^{\prime}\right)=\varphi\left(P_{N} u\left(t^{\prime}\right), t^{\prime}\right)$, то из единственности решения задачи $(0.1),(1.4)$ следует, что $Q_{N} u(t)=\varphi\left(P_{N} u(t), t\right) \forall t \geqslant t^{\prime}$. Тогда $(1.7)$ выполнено при $t_{1}=t^{\prime}$,

$$
v(t)=p\left(t ; \varphi, P_{N} u^{\prime}\left(t_{1}\right), t_{1}\right)+\varphi\left(p\left(t ; \varphi, P_{N} u^{\prime}\left(t_{1}\right), t_{1}\right), t\right) .
$$

Пусть для любых $t \geqslant \tau+T$ выполнено $Q_{N} u(t) \neq \varphi\left(P_{N} u(t), t\right)$. Обозначим $s=\tau+T$. $\Phi$ ункция $u(t)$ при $t>s$ удовлетворяет уравнению $(0.1)$ с начальным условием $\left.u\right|_{t=s}=u(s)$.

ПРЕДЛОЖЕНИЕ 2.1. Существует по крайней мере одна точка $p_{0} \in P_{N} V$ такая, что если

$$
\left\|\varphi\left(p_{0}, s\right)-Q_{N} u(s)\right\|_{\beta}>\left\|p_{0}-P_{N} u(s)\right\|_{\beta},
$$

mo

$$
\left\|\varphi\left(p\left(t ; \varphi, p_{0}, s\right), t\right)-Q_{N} u(t)\right\|_{\beta}>\left\|p\left(t ; \varphi, p_{0}, s\right)-P_{N} u(t)\right\|_{\beta} \quad \forall t>s .
$$

Обозначим через $p_{0}^{*}$ точку, для которой выполнены неравенства (2.9) и (2.10). Положим $v(t)=p\left(t ; \varphi, p_{0}^{*}, s\right)+\varphi\left(p\left(t ; \varphi, p_{0}^{*}, s\right), t\right)$. Используя $(2.10)$, получим, что для любых $t \geqslant s$ верно неравенство

$$
\|v(t)-u(t)\|_{\beta} \leqslant 2\left\|Q_{N}(v(t)-u(t))\right\|_{\beta} .
$$

Согласно сжимающему свойству (см. [3]) при выполнении условия (2.10) справедливо неравенство

$$
\left\|Q_{N}(v(t)-u(t))\right\|_{\beta} \leqslant\left\|Q_{N}(v(s)-u(s))\right\|_{\beta} e^{-\mu_{N}(t-s)} .
$$

Из (2.11) и (2.12) следует (1.7). Теорема доказана. 
3. Уравнение реакции диффузии. В этом пункте построено интегральное многообразие задачи

$$
\begin{aligned}
& u_{t}-u_{x x}+f(u, x, t)=0, \quad x \in \Omega=[0, \pi] \\
& \left.u\right|_{t=\tau}=u_{\tau},\left.\quad u\right|_{\partial \Omega}=0 .
\end{aligned}
$$

Обозначим

$$
F(v, x, t)=\int_{0}^{v} f(\omega, x, t) d \omega .
$$

Предположим, что функции $f(v, x, t)$ и $F(v, x, t)$ удовлетворяют следующим условиям:

$$
\begin{gathered}
f v \geqslant-K_{1} v^{2}-K_{2}, \quad \text { где } 1-K_{1} \pi=\alpha>0, \\
f \in C^{3}\left(\mathbb{R}^{3}\right), \\
F_{t}(v, x, t) \leqslant K_{3} F(v, x, t)+K_{4}, \quad v, t \in \mathbb{R}, \quad x \in \Omega, \\
-K_{7} \leqslant F(v, x, t) \leqslant K_{5}^{-1} f(v, x, t) v+K_{6}, \quad v, t \in \mathbb{R}, \quad x \in \Omega, \\
\left|\frac{\partial^{i}}{\partial x^{i}}\left(f\left(v, x, t_{1}\right)-f\left(v, x, t_{2}\right)\right)\right| \leqslant M_{i}(|u|)\left|t_{1}-t_{2}\right|, \quad i=0,1,2, \quad t_{1}, t_{2}, v \in \mathbb{R}, \quad x \in \Omega .
\end{gathered}
$$

В этих формулах $K_{i}>0, i=1, \ldots, 7,-$ некоторые константы, $M_{i}(\cdot), i=0,1,2,-$ монотонные функции.

Лемма 3.1. Пусть выполнены условия (3.3)-(3.7). Тогда существует единственное решение $u(t) \in C_{b}\left(\mathbb{R}_{\tau}, H^{2}(\Omega)\right)$ задачи (3.1), (3.2). Задача (3.1), (3.2) обладает ограниченным в $H^{2}(\Omega)$ поглощающим множеством.

По формуле (1.5) определим срезанную функцию $F$ и рассмотрим измененное уравнение

$$
u_{t}-u_{x x}+F(u, x, t)=0 .
$$

ТЕОрема 3.1. Пусть выполнены условия (3.3)-(3.7). Тогда уравнение (3.8) обладает интегральным многообразием, которое әкспоненииально притягивает в пространстве $H^{1}(\Omega)$ все решения задачи (3.1), (3.2).

Доказательство следует из общей теоремы 1.1.

Московский государственный университет

Поступило им. М.В. Ломоносова

26.09 .96

\section{СПИСОК ЦИТИРОВАННОЙ ЛИТЕРАТУРЫ}

1. Eden A., Foias C., Nicolaenko B., Temam R. Inertial Sets for Dissipative Evolution Equations. Preprint Series 812, 1991. 2. Foias C., Sell G. R., Temam R. // J. Differential Equations. 1988. V. 73. № 2. 3. Foias C., Sell G. R., Titi E. Exponential Tracking and Approximation of Inertial Manifolds for Dissipative Nonlinear Equations. Preprint 8803: Indiana Univ.. 4. Чуешов И.Д. Введение в теорию инерциальных многообразий. Харьков: ХГУ, 1992. 5. Бабин А. В., Вишик М. И. Аттракторы эволюционных уравнений. М.: Наука, 1989. 